\title{
A Robust Electrode Configuration for Bioimpedance Measurement of Respiration
}

\author{
Hong-bin Wang1, Chen-wen Yen ${ }^{2}$, Jing-tao Liang ${ }^{1}$, Qian Wang ${ }^{1}$, \\ Guan-zheng Liu ${ }^{1}$ and Rong Song ${ }^{1 *}$ \\ ${ }^{1}$ School of Engineering, Sun Yat-sen University, Guangzhou, \\ People's Republic of China \\ ${ }^{2}$ Department of Mechanical and Electro-mechanical Engineering, \\ National Sun Yat-Sen University, Kaohsiung, Taiwan
}

\begin{abstract}
Electrode configuration is an important issue in the continuous measurement of respiration using impedance pneumography (IP). The robust configuration is usually confirmed by comparing the amplitude of the IP signals acquired with different electrode configurations, while the relative change in waveform and the effects of body posture and respiratory pattern are ignored. In this study, the IP signals and respiratory volume are simultaneously acquired from 8 healthy subjects in supine, left lying, right lying and prone postures, and the subjects are asked to perform four respiratory patterns including free breathing, thoracic breathing, abdominal breathing and apnea. The IP signals are acquired with four different chest electrode configurations, and the volume are measured using pneumotachograph (PNT). Differences in correlation and absolute deviation between the IP-derived and PNT-derived respiratory volume are assessed. The influences of noise, respiratory pattern and body posture on the IP signals of different configurations have significant difference $(\mathrm{p}<0.05)$. The robust electrode configuration is found on the axillary midline, which is suitable for long term respiration monitoring.
\end{abstract}

Keywords: Impedance pneumography, respiratory volume, electrode configurations, respiration, pneumotachograph

\section{INTRODUCTION}

Noninvasive bioelectrical impedance measurement has been widely implemented for long-term monitoring of thoracic impedance. Impedance pneumography (IP) signals can be used to assess respiration variables such as tidal volume and respiration rate [12]. The IP signal is usually acquired through electrodes placed on the thorax, and different electrode configurations show significant difference in amplitude and waveform [3]. Previous studies sought the robust configuration based on the amplitude of the IP signals acquired from different electrode configurations, while the relative change in waveform and the effects of body posture and respiratory pattern are ignored [3-4]. Respiratory volume has important applications on detecting asthma, chronic

*Corresponding author: Rong Song, School of Engineering, Sun Yat-sen University, Guangzhou, Peoples R China. Phone: (86) 020-39332148, E-mail: songrong@mail.sysu.edu.cn. Other authors: wanghbin@mail2.sysu.edu.cn; cmu robot@gmail.com; 604314584@qq.com; wangqian.99@163.com; liugzh3@mail.sysu.edu.cn. 
obstructive pulmonary disease, cystic fibrosis, and airway obstruction disease through the analysis of various respiratory parameters [5-10], and it is usually measured in clinic using a pneumotachograph (PNT) or a respiratory flow meter, but these devices may increase mechanical impedance and psychological load, which may affect subjects' normal respiratory pattern. At the same time, they are not suitable for long-term monitoring due to respiratory dead space [9]. Bioelectrical impedance measurement could effectively avoid these problems and enable long-term monitoring in clinic or daily life.

Since all the measurements are performed through the electrode system, a robust electrode configuration is essential for accurate respiratory volume measurement. However, insufficient attention has been devoted to the electrode configuration [11-12]. Lanat et al. applied a wearable impedance measurement system, called wealthy shirt, to acquire the IP signal through electrodes on the left and right sides of nipples, and was compared with the respiratory signals separately acquired with flow meter, inductive plethysmography, piezoresistive textile sensors and piezoelectric sensors. The results indicated that respiratory rate could be detected well from the IP signal, but the signal was susceptible to motion artifacts [13]. Lahtinen et al. demonstrated that the IP signals acquired from running subjects with different electrode configurations were affected differently by noise, and the configuration with the least motion artifact interference varied with different depth of breathing and respiratory patterns [14]. Koivumäki et al. extracted the IP and impedance cardiography (ICG) signals from thoracic impedance signals, and the measuring electrodes were placed on the left and right thoracic axillary midline. The IP and ICG signals were separately compared with PNT and electrocardiograph (ECG) signals, and the results indicated that respiratory rate, heart rate and time interval between the IP and ICG signals could be derived from the impedance signals [15].

Several studies have documented relationship between the respiratory movements of thorax and the airflow signals. Carry et al. compared the respiratory inductive plethysmography (RIP) signal with the PNT signal and concluded that the RIP signal seemed to represent the respiratory waveform reasonably well with subjects breathing against a resistive load [16]. Eberhard et al. documented consistency between the airflow and RIP signals in three different postures (seated, lateral and dorsal supine) while the subjects were in controlled resistive loaded conditions [17]. Seppä et al. discussed five different electrode configurations distributed over thorax for continuous long-term measurement of tidal breathing in mobile subjects, and the results showed that the agreement in the respiratory flow signals derived from the IP and PNT signals were excellent and the waveform of the PNT-derived respiratory volume was very similar to that derived from IP signals, and the main source of error in the IP signals was the cardiogenic distortion [18]. All the above-mentioned researchers have studied the application of impedance pneumography to the measurement of respiration, but different electrode configurations are used in their experiments.

The purpose of this study is to validate a robust electrode configuration suitable for long-term bioimpedance measurement of respiration based on relative and absolute waveform similarity between the IP-derived and PNT-derived respiratory volume. In 
addition, the effects of noise, respiratory pattern and body posture on IP signal are addressed.

\section{MATERIALS AND METHODS}

\subsection{Test Subjects and Measurement System}

Eight healthy male students volunteered as study subjects. They ranged in age from 22 to 26 years old, in body mass index from 19 to $30 \mathrm{~kg} / \mathrm{m}^{2}$, and in chest size from $80-115$ $\mathrm{cm}$. None of them suffer from any respiratory disease. They did not receive any remuneration or other benefits for participating, and each participant gave written informed consent. The study was approved by the ethics committee of the sixth affiliated hospital of Sun Yat-sen university.

The IP signal is acquired with the sample frequency of $100 \mathrm{~Hz}$ through an impedance meter (E4980A, Agilent, USA) which could provide current with 0-2 mA in amplitude and $0-2 \mathrm{MHz}$ in frequency, and the resolution of the impedance meter is $0.0001 \Omega$ when the measured impedance is smaller than $100 \Omega$. The amplitude and frequency of the stimulus current used in this experiment are $500 \mu \mathrm{A}$ and $50 \mathrm{k} \mathrm{Hz}$ [13]. The single impedance measurement channel is converted to four channels through a custom-made multi-way switch converter. The switch converter is composed of a micro controller unit (MCU) and a digital controlled analog switch, and the analog switch is controlled by the MCU with $5 \mu \mathrm{s}$ switching time. Common ECG electrodes with $1.5 \mathrm{~cm}$ in diameter (Ag/Agcl electrodes, Shanghai Jun Kang Medical Equipment Company, China) are used to supply the stimulus current and detect any voltage change. The measured impedance values are transmitted through the general purpose interface bus (GPIB) to a computer for display and storage.

The PNT signal is measured using a respiratory flow meter (TSD117, Biopac, USA) with the sample frequecny of $100 \mathrm{~Hz}$. The data are amplified, filtered and then transmitted through the network interface to the computer for further processing. The respiratory flow meter is calibrated using a $3.00 \mathrm{~L}$ calibration cylinder. When the error of the volume values exceed $\pm 1 \%$, the system is recalibrated. All the measurement devices are controlled by a synchronization control signal from the computer, and the block diagram of the measurement system is shown in Figure 1.

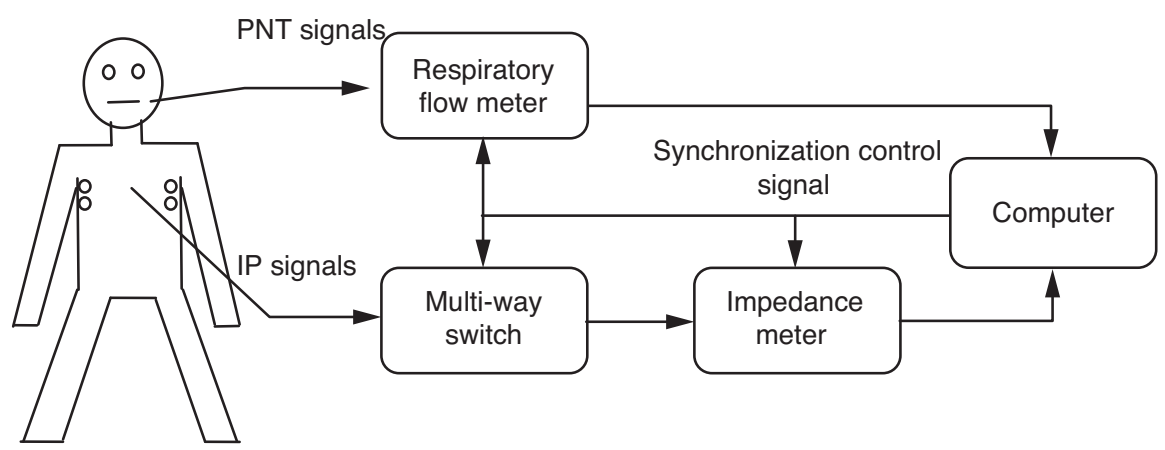

Figure 1. Block diagram of the measurement system 


\subsection{Electrode Placement}

In four-electrode measurement, a constant stimulus current is discharged through one pair of electrodes, and voltage reflecting changes of tissue conductivity can be detected by the other pair of electrodes [19]. The four-electrode measurement has advantages over the two-electrode method in terms of minimizing the effect of contact impedance between skin and electrodes [18]. Based on previous studies [13-15, 18, 20], 4 typical thoracic electrode configurations are selected for acquiring the IP signals in this study. The positions of electrodes are shown in Figure 2 and Table 1.

(A)

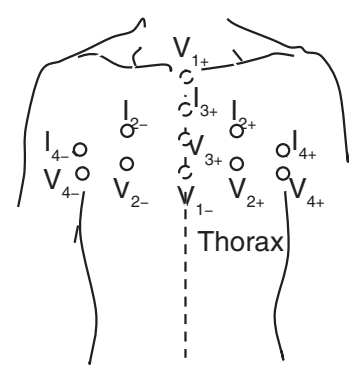

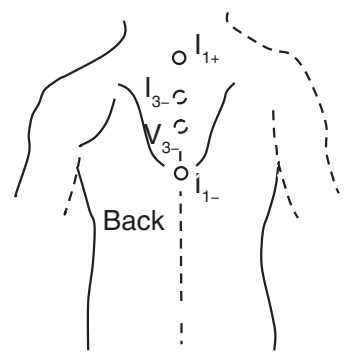

(B)

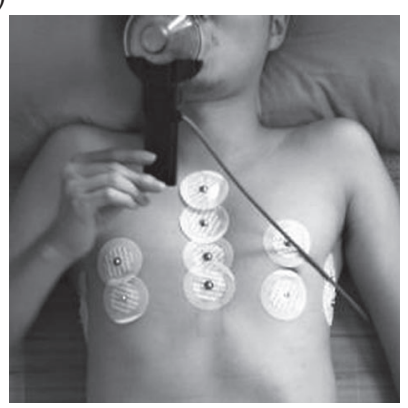

Figure 2. (A) The electrode configurations; (B) the photo of electrode positions. All the configurations are used in one experiment.

Table 1. Electrode positioning details.

\begin{tabular}{|c|c|c|}
\hline $\begin{array}{l}\text { Configuration } \\
\text { code }\end{array}$ & $\begin{array}{l}\text { Electrode } \\
\text { codes }\end{array}$ & Physical locations \\
\hline A & $\mathrm{I}_{1+} \mathrm{V}_{1+} \mathrm{V}_{1-} \mathrm{I}_{1-}$ & $\begin{array}{l}\mathrm{V}_{1+} \text { on the sternum, } \mathrm{V}_{1-} \text { at the xiphoid locations; } \\
\mathrm{I}_{1+} \text { and } \mathrm{I}_{1-} \text { on the back over the spine } 2 \mathrm{~cm} \\
\text { above and below } \mathrm{V}_{1+} \text { and } \mathrm{V}_{1-\text { - }} \text {, respectively. }\end{array}$ \\
\hline B & $\mathrm{I}_{2+} \mathrm{V}_{2+} \mathrm{V}_{2-} \mathrm{I}_{2-}$ & $\begin{array}{l}\mathrm{V}_{2+} \text { and } \mathrm{V}_{2-} 2 \mathrm{~cm} \text { below the nipples; } \\
\mathrm{I}_{2+} \text { and } \mathrm{I}_{2-} 2 \mathrm{~cm} \text { above the nipples, and } \\
\text { the } \mathrm{I}_{2+} \text { and } \mathrm{V}_{2+} \text { on the left side of thorax. }\end{array}$ \\
\hline $\mathrm{C}$ & $\mathrm{I}_{3+} \mathrm{V}_{3+} \mathrm{V}_{3-} \mathrm{I}_{3-}$ & $\begin{array}{l}I_{3+} \text { and } V_{3+} \text { on the average manubrium between } \\
\text { the sternum and the xiphoid; } I_{3-} \text { and } V_{3-} \text { at the } \\
I_{3+} \text { and } V_{3+} \text { locations projected on the back. }\end{array}$ \\
\hline D & $\mathrm{I}_{4+} \mathrm{V}_{4+} \mathrm{V}_{4-} \mathrm{I}_{4-}$ & $\begin{array}{l}\mathrm{I}_{4+} \text { and } \mathrm{V}_{4+} \text { on the intersections of left axillary } \\
\text { midline with the fifth and seventh ribs; } \\
\mathrm{I}_{4-} \text { and } \mathrm{V}_{4-} \text { on the intersections of right axillary } \\
\text { midline with the fifth and seventh ribs }\end{array}$ \\
\hline
\end{tabular}

I: stimulus electrode. V: voltage measuring electrode. 


\subsection{Measurement Procedure}

All electrodes are first affixed in the configurations, and the subjects are then instructed to wear a nose clip and lie in an appropriate test position with left or right hand holding the flow meter, as shown in Figure 2(B).

Subjects are asked to adopt four respiratory patterns: free breathing (FB), thoracic breathing (TB), abdominal breathing (AB) and apnea. The sequence is shown in Figure 3, including FB for 2 min, TB for 2 min, FB for 1 min, AB for 2 min, FB for $1 \mathrm{~min}$ and apnea for 30 seconds. Each subject is instructed to change respiratory pattern following the same sequence shown in Figure 3, while the subject lies in bed supine, left lying, right lying and prone. For TB state, the subjects are instructed to breathe normally using only the thoracic muscles and minimizing the movement of abdomen. For $\mathrm{AB}$ state, the subjects are asked to breathe normally using only the abdominal muscles and minimizing the movement of thorax [4]. The purpose of TB and AB is to study the effect of respiratory patterns on the IP signals acquired from different electrode configurations. The purpose of FB is mainly to regulate the breathing state. The IP signal is acquired as the noise while the subjects are in temporary apnea.

\subsection{Signal Processing}

The IP and PNT signals are first processed by a fourth-order band-pass Butterworth filter with a frequency band of $0.05-10 \mathrm{~Hz}$, which could effectively remove baseline drift, fluctuations and high-frequency interference from the ECG and electromyography signals [18]. The processed IP signal containes some noise, such as blood flow, whose frequency spectrum overlaps with that of the respiratory signal. The noise could not be ignored due to the fact that it has a little impact on the waveform of the IP signal. To study the interference of other physiological signals on the IP signal, frequency spectrum integrals (FSI) which are the spectrum integral value of IP signal from 0.05 $10 \mathrm{~Hz}$, are calculated while the subjects perform FB, TB, AB and apnea in all the body postures. When the subjects are in apnea state, the IP signal mainly represents the physiological crosstalk. While the subjects are in $\mathrm{FB}, \mathrm{TB}$ and $\mathrm{AB}$ states, the physiological crosstalk is part of the IP signal. The noise ratio (NR) between FSI of $\mathrm{IP}_{\text {apnea }}$ and IP signals represents the impact of the physiological crosstalk on the IP signal, and is calculated as:

$$
N R=\frac{F S I\left(I P_{\text {apnea }}\right)}{F S I(I P)}
$$

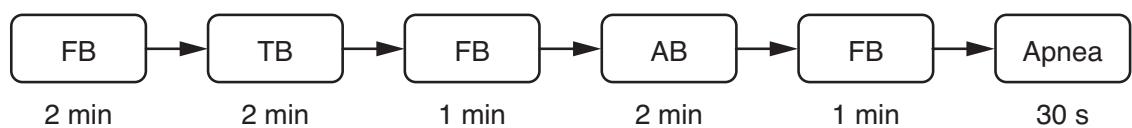

Figure 3. The sequence of respiratory phases. (FB: free breathing; TB: thoracic breathing; AB: abdominal breathing). 
The filtered IP and integrated PNT (IPNT) signals are divided into segments corresponding to the recorded start and end times of each respiratory phase. For each breathing segment, the Pearson correlation index (CI) between the IP and IPNT signals is calculated. The segmented IP and IPNT signals are then again equally divided into two parts. For one part of the IP and IPNT signals, the calibration parameters are achieved by computing the mean values of the ratios between every point of the IPNT and IP signals. The other section of the IP signal is calibrated by multiplying the calibration parameters, and the root mean square error (RMSE) between the calibrated IP and IPNT signals is calculated. The agreement between tidal volume of IP signal (TVip) and tidal volume of IPNT signal (TVipnt) is then analyzed, where TVip and TVipnt are the difference between the maximum and minimum values of the calibrated IP and IPNT signals, respectively.

Finally, the NR values are tested by paired t-test, and the tidal volume values are analyzed by correlation and Bland-Altman analysis. Three-way ANOVA is used to analyze the effects of all the factors (electrode configuration, respiratory pattern, body posture) on CI and RMSE [21], and the effects of respiratory pattern and body posture are further studied using two-way ANOVA.

\section{RESULTS}

The typical processed IP, PNT and IPNT signals are shown in Figure 4. The IP signal represents the impedance change of thorax caused by the lung ventilation, and the PNT signal reflects the respiratory airflow rate. The IP and PNT signals are out of phase, and the amplitudes of the IP signals of all the configurations are different from each other. The IPNT signal reflects the respiratory volume, and the phase and waveform of the IP signal show good consistency with the IPNT signal, which are consistent with other studies [22].

(A)

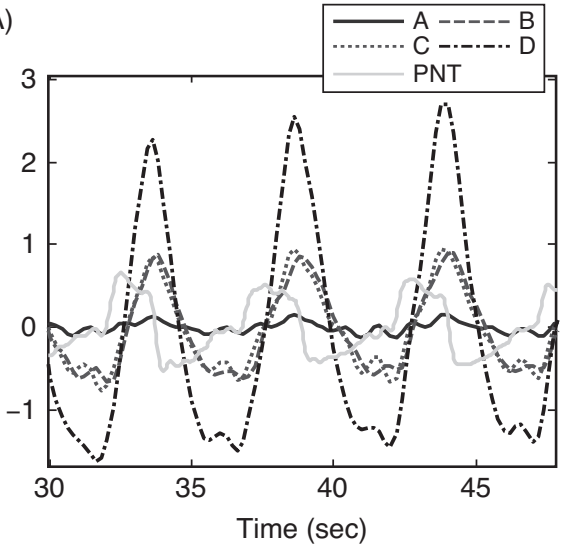

(B)

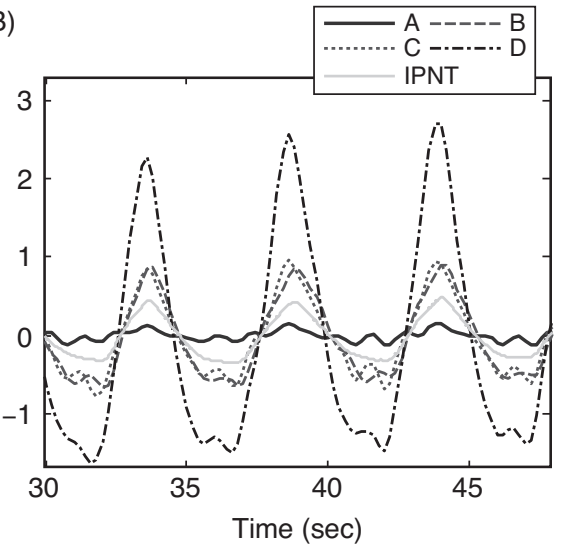

Figure 4. (A) An example of the IP and PNT signals with the subject in free breathing and supine posture; (B) An example of the IP and IPNT signals with the subject in free breathing and supine posture. A, B, C and D represent the IP signals in $\Omega$ acquired from 4 different configurations; PNT and IPNT reflect respiratory flow in litre per second and volume in litre, respectively. 


\subsection{The Effects of Noise}

The NR values of the IP signals for all body postures and respiratory patterns are shown in Figure 5, and the results of paired t-test of all the configurations are listed in Table 2. The NR value decreases following the order from configuration A toward D. In most of the paired comparisons, the NR values of the IP signal in electrode configuration $\mathrm{D}$ are significantly lower than those in configurations A, B and C $(p<0.05)$.

\subsection{Effects of Electrode Configurations}

The CI and RMSE values between the IP and IPNT signals of all the measurement samples are summarized in Figure 6. For configurations B, C and D, the CI values are close to 1 while the subjects are in $\mathrm{AB}$ state, and the $\mathrm{CI}$ values for the subjects in the $\mathrm{FB}$ and $\mathrm{TB}$ states are smaller than those in $\mathrm{AB}$ state in most of body postures. Configuration D is generally best with mean $\mathrm{CI}$ values between 0.95 and 1.00 .
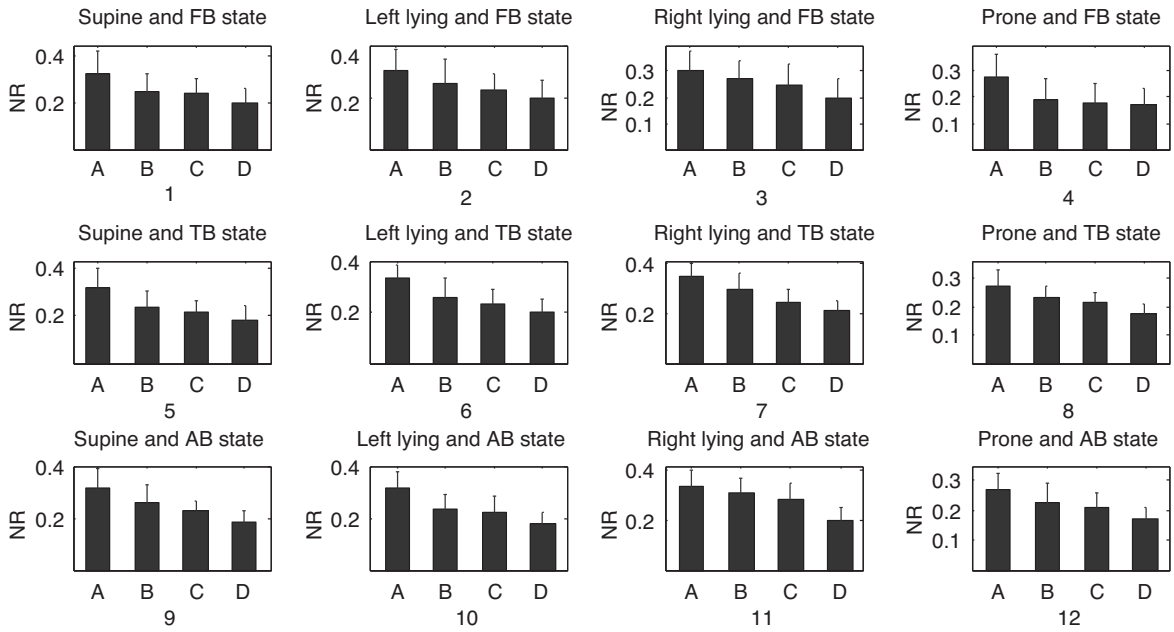

Figure 5. The NR values for all configurations in all body postures and respiratory patterns.

Table 2. The results of paired t-test of all the configurations. (The numbers 1 to 12 correspond to those in Figure 5).

\begin{tabular}{ccccccccccccc}
\hline & $\mathbf{1}$ & $\mathbf{2}$ & $\mathbf{3}$ & $\mathbf{4}$ & $\mathbf{5}$ & $\mathbf{6}$ & $\mathbf{7}$ & $\mathbf{8}$ & $\mathbf{9}$ & $\mathbf{1 0}$ & $\mathbf{1 1}$ & $\mathbf{1 2}$ \\
\hline A\&B & 0.02 & 0.00 & 0.00 & 0.00 & 0.00 & 0.01 & 0.00 & 0.17 & 0.03 & 0.00 & 0.09 & 0.02 \\
A\&C & 0.01 & 0.01 & 0.01 & 0.01 & 0.00 & 0.00 & 0.00 & 0.04 & 0.00 & 0.00 & 0.10 & 0.01 \\
A\&D & 0.00 & 0.00 & 0.00 & 0.01 & 0.00 & 0.00 & 0.00 & 0.01 & 0.00 & 0.00 & 0.00 & 0.00 \\
B\&C & 0.39 & 0.20 & 0.16 & 0.68 & 0.13 & 0.26 & 0.01 & 0.02 & 0.09 & 0.34 & 0.46 & 0.28 \\
B\&D & 0.03 & 0.03 & 0.00 & 0.48 & 0.01 & 0.04 & 0.00 & 0.00 & 0.00 & 0.00 & 0.01 & 0.03 \\
C\&D & 0.08 & 0.03 & 0.00 & 0.59 & 0.01 & 0.09 & 0.03 & 0.01 & 0.01 & 0.00 & 0.00 & 0.00 \\
\hline
\end{tabular}


(A)

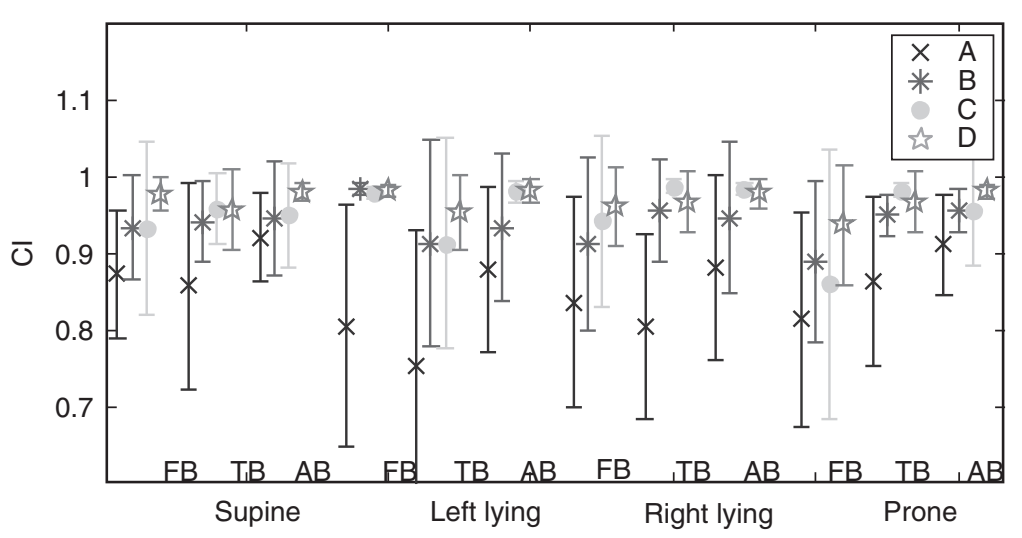

Body posture

(B)

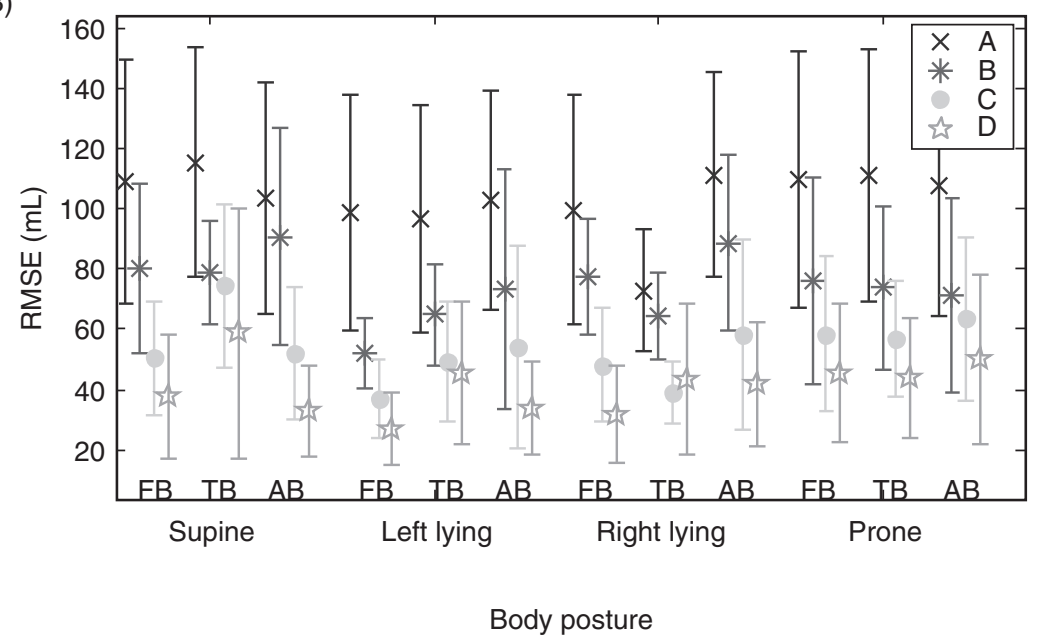

Figure 6. (A) The CI values between the IP and IPNT signals in various respiratory patterns and body postures. (B) The RMSE values between the IP and IPNT signals in various respiratory patterns and body postures. (The central points mark the mean values of CI and RMSE of all the subjects, and vertical lines represent the standard deviation).

Configuration A $(0.7<$ mean $\mathrm{CI}<0.9)$ always produces CI smaller than those of configurations B, C and D. Figure 6(B) shows that the RMSE value decreases in the order from configurations A toward D except when the subjects perform TB in right lying. For configuration D, the RMSE value increases when the subjects perform TB in supine, left lying and right lying postures, while the smallest RMSE values in prone state are observed when the subjects perform TB. 
Table 3. The results of three-way ANOVA of CI and RMSE. $\left({ }^{*} p<0.05\right)$

\begin{tabular}{lcc}
\hline Source & Sig. of CI & Sig. of RMSE \\
\hline Electrode configuration & $0.000^{*}$ & $0.000^{*}$ \\
Respiratory pattern & $0.004^{*}$ & 0.235 \\
Body posture & 0.637 & $0.006^{*}$ \\
Electrode configuration vs. Respiratory pattern & 0.181 & 0.476 \\
Electrode configuration vs. Body posture & 0.358 & 0.879 \\
Respiratory pattern vs. Body posture & $0.015^{*}$ & 0.054 \\
Electrode configuration vs. Respiratory pattern vs. Body posture & 0.999 & 0.986 \\
\hline
\end{tabular}

All the data are tested for normality with Kolmogorov-Smirnov test (sig. of $\mathrm{CI}=0.063>0.05$, sig. of RMSE $=0.506>0.05$ ), and three-way ANOVA analysis is adopted to investigate the effects of different factors (electrode configuration, respiratory pattern and body posture). In Table 3, the results indicate that the electrode configuration is significantly related to both the CI and RMSE, and both the effects of respiratory pattern on the CI and effects of body posture on the RMSE are significant, while the effects of respiratory pattern on the RMSE and effects of body posture on the CI are not significant. Regarding the interactions of the three factors, only the interaction of respiratory pattern with body posture is significantly related to the CI. The other combinations of electrode configuration, respiratory pattern and body posture show no significant effects on RMSE or CI.

\subsection{Effects of Respiratory Pattern and Body Posture}

Figure 7(A) presents the mean CI values with different configurations and respiratory patterns. In all four electrode configurations, the CI value clearly varies with respiratory pattern, and the CI values of configurations $\mathrm{B}$ and $\mathrm{D}$ demonstrate smaller variations with the change of respiratory pattern than those of configurations $\mathrm{A}$ and $\mathrm{C}$. The CI values of configuration $\mathrm{D}$ are greater than 0.95 in all the respiratory patterns, and the CI values of configuration $\mathrm{A}, \mathrm{B}$ and $\mathrm{C}$ are less than those of configuration $\mathrm{D}$. The mean $\mathrm{CI}$ values for all the cofigurations in various body postures are shown in Figure 7(B). The CI value increases following the order from configuration A toward D in all postures. The effects of posture vary with the electrode configurations. For configuration $\mathrm{D}$, the $\mathrm{CI}$ values are almost the same for all the postures. However, the CI value of configuration A changes markedly with postures. Two-way ANOVA reveals that the CI values of all the configurations are related to respiratory pattern and body posture, and the CI values of configuration A are significantly smaller than those of the other configurations $(p<0.05)$, and the CI values of configurations $\mathrm{B}, \mathrm{C}$ and $\mathrm{D}$ do not show any significant difference among one another.

The mean RMSE values of all the configurations caused by respiratory pattern are shown in Figure 7(C). The RMSE values of configurations C and D increase when the subjects practice TB, and the RMSE values of configurations A and B decrease in TB state. Two-way ANOVA reveals that the RMSE values of 
(A)

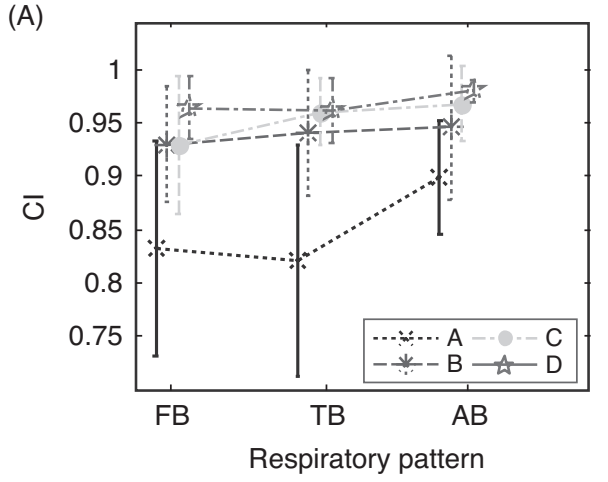

(C)

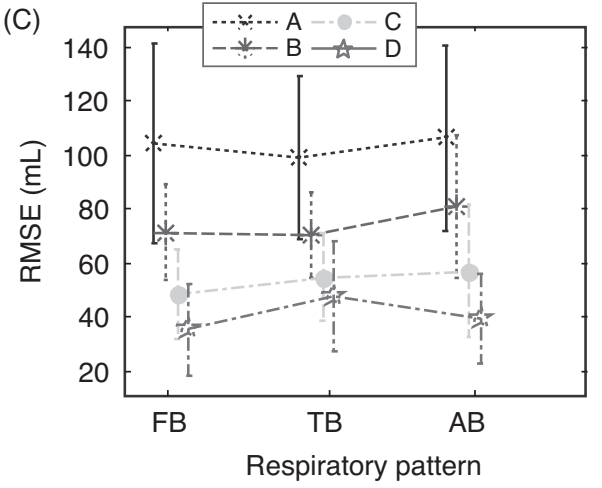

(B)

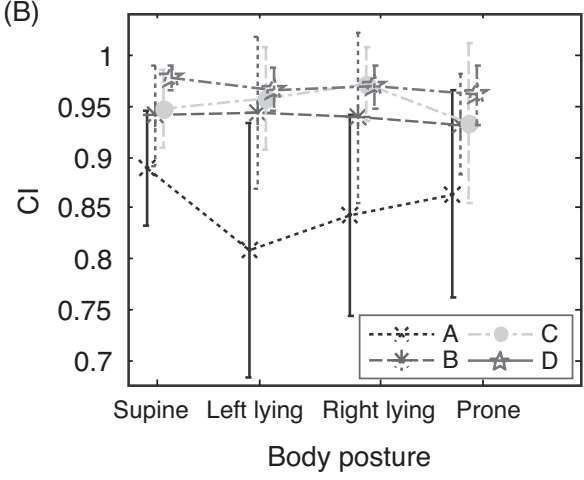

(D)

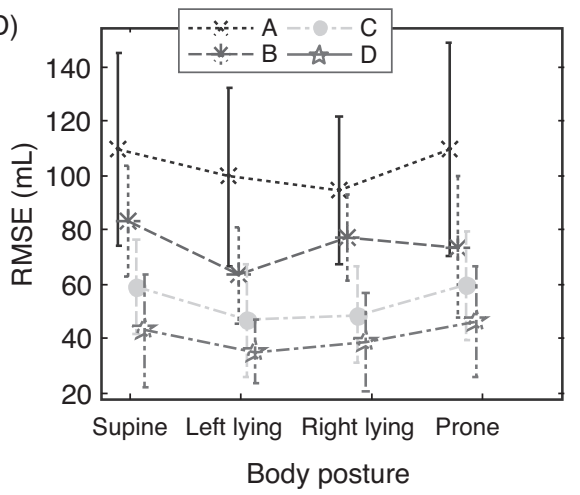

Figure 7. (A) CI values for different respiratory patterns. (B) CI values for different body postures. (C) RMSE values for different respiratory patterns. (D) RMSE values for different body postures. (The central points mark the mean values of CI and RMSE of all the subjects, and vertical lines represent the standard deviation).

configuration D are significantly smaller than those of configurations A and B, while there is no significant difference between the RMSE values of configurations $\mathrm{C}$ and D ( $\mathrm{p}>0.05)$. Figure 7(D) displays the mean RMSE values related to postures. The variation of RMSE value in configuration D with body postures is smaller than those of other configurations. Two-way ANOVA shows that the RMSE values of configuration $\mathrm{D}$ are significantly smaller than those of configurations $\mathrm{A}$, $\mathrm{B}$ and $\mathrm{C}$ in all the body postures $(\mathrm{p}<0.05)$.

\subsection{Tidal Volume Agreement}

The relationship between TVip and TVipnt is investigated, and the results are shown in Figure 8. The $\mathrm{r}^{2}$ value increases following the order from configuration A toward $\mathrm{D}$, and all values are greater than 0.9 . The high linear correlation between TVip and TVipnt indicates that the IP signal could well reflect the tidal volume. 
(A)

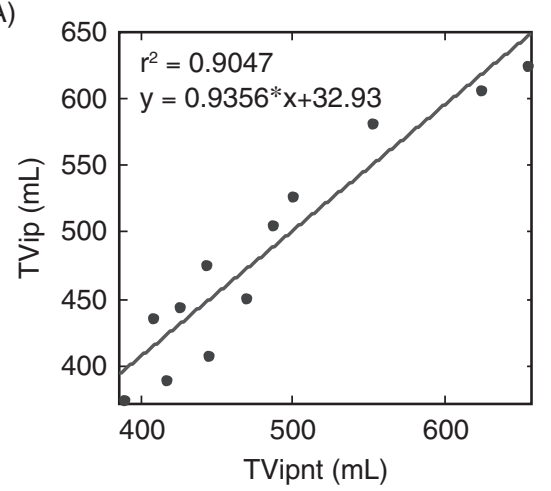

(C)

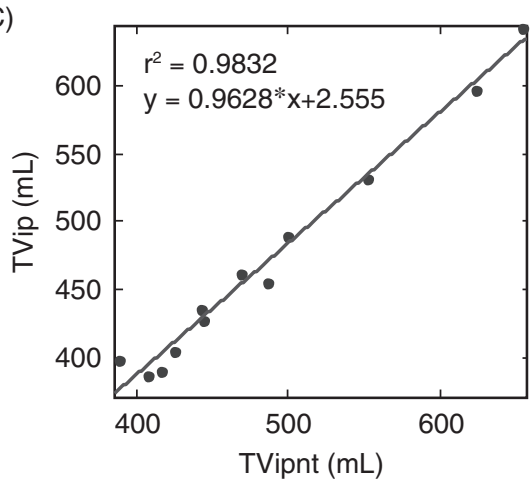

(B)

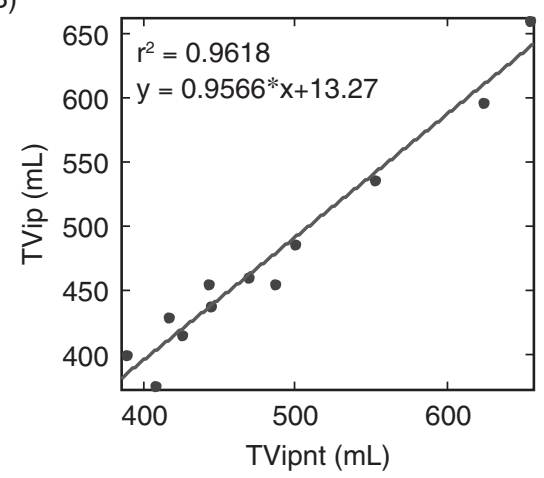

(D)

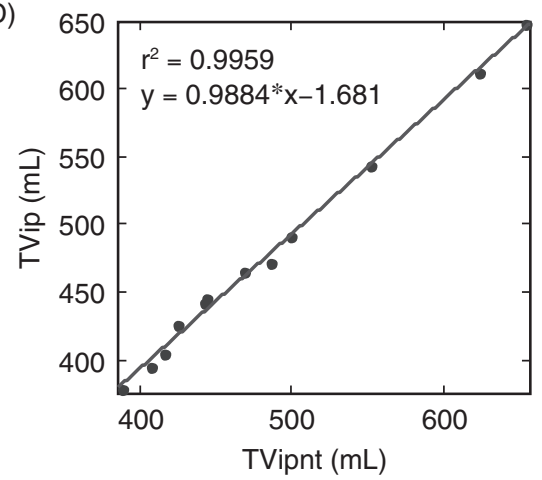

Figure 8. TVip vs. TVipnt for configurations A, B, C, and D.

The results of Bland-Altman analysis between TVip and TVipnt is presented in Figure 9. The mean value of difference between TVip and TVipnt increases following the order of configurations A, D, B and C, suggesting that the system bias between configurations $\mathrm{A}$ and $\mathrm{D}$ is relatively small. While the largest scattering range of configuration A indicates bad agreement between TVip and TVipnt, the smallest scattering range of configuration D indicates that TVip has the best agreement with TVipnt among all the configurations.

\section{DISCUSSION}

\subsection{The Effects of Noise}

The movements of thoracic muscles disappear when the subjects are in apnea, leaving blood flow as the main source of noise in all configurations [23]. The waveform distortions of the IP signal are mainly caused by noise, and the robust electrode configuration is expected to minimize the impact of noise. The smaller NR values of configuration D in most of respiratory patterns and body postures show its robustness to noise. 
(A)

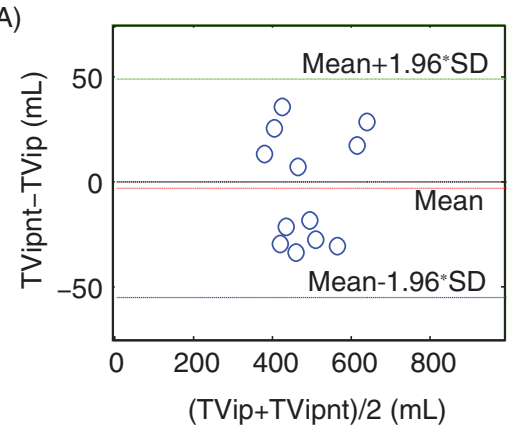

(C)

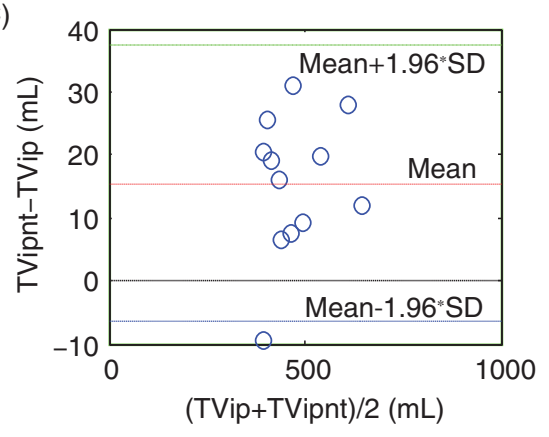

(B)

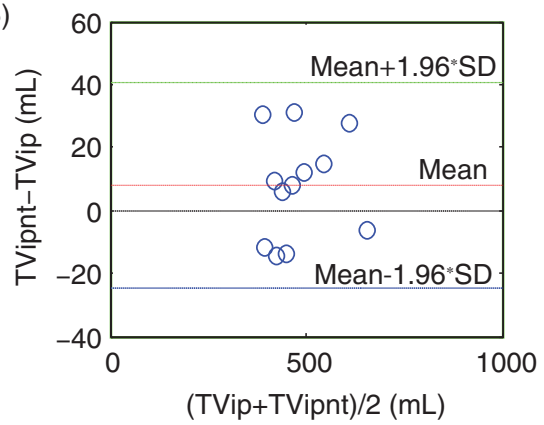

(D)

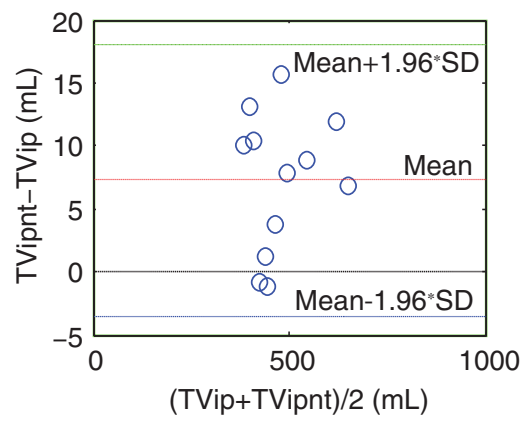

Figure 9. Bland-Altman analysis between TVip and TVipnt for configurations A, $\mathrm{B}, \mathrm{C}$ and D. (Each point is calculated from a combination of different respiratory pattern and body posture. Mean represents the mean value of the differences between tidal volumes derived from the IP and IPNT signals; mean $\pm 1.96 * \mathrm{SD}$ represents $95 \%$ limits of agreement).

\subsection{CI and RMSE}

The IP and IPNT signals are in different units and have different amplitudes; Pearson correlation index is used to investigate the relative waveform difference between the IP and IPNT signals [24-25]. The RMSE values are computed to assess the absolute waveform deviation between the calibrated IP and IPNT signals because RMSE is sensitive to measurement error in the IP signal [26-27]. CI proves insensitive to body posture while RMSE is relatively more sensitive. Respiratory pattern, by contrast, has greater impact on CI than on RMSE. The combination of CI and RMSE assists in defining the robust electrode configuration, which are least affected by respiratory pattern and body posture.

Configuration D in general yields the best CI and RMSE values between the IP and IPNT signals. In most conditions, with different respiratory patterns, the trend of CI is opposite to that of RMSE for configuration D. Only with the subjects in prone posture, RMSE and CI of configuration D have the same trend, and this might be due to calibration errors. The impedance-volume calibration parameters in these experiments are the mean of the ratios between the IPNT and IP signals at every sampling point. The 
method is susceptible to interference, and the posture and breathing pattern should be specified to possibly make the calibration more reliable [27-28].

\subsection{Electrode Configuration}

All the results indicate that the IP signal acquired from configuration D using four-electrode method well represents the IPNT signal and is least affected by noise. The robust configuration is different from the configurations of some studies due to different experimental protocols and evaluation parameters [29-31]. In this study, the robust electrode configuration is tested in a more comprehensive way, which not only adopts the absolute and relative evaluation parameters, but also confirms the performance in various respiratory patterns and body postures. Similar electrode locations were also tested in previous works $[18,31]$ that showed configuration $\mathrm{D}$ had the highest volume measurement sensitivity, consistent with the result of this study. For configuration D, the small RMSE values between the calibrated IP and IPNT signals for all the respiratory patterns and body postures tested show that the IP signal could well reflect the respiratory volume, and the tidal volume value derived from IP signal is in good agreement with that derived from IPNT signal. Incomplete respiratory volume is acquired from configurations $\mathrm{A}, \mathrm{B}$ and $\mathrm{C}$, as reflected by smaller amplitudes of IP signals. The electrodes on the left and right axillary midline in configuration D are far from the central zone of chest, and the respiratory movements have less influence on the contact surface artifacts between electrodes and the skin. The electrodes in configurations A, B and C are near the central zone of chest, and the IP signal is susceptible to the contact surface artifacts caused by the respiratory movements; therefore, configurations A, B and C seem particularly unsuitable for long-term respiration monitoring. Different body postures cause different degrees of thorax deformation, affecting the electric field distribution sensed by the electrodes [32]. With configurations A, B and C, the impacts of thorax volume deformation and the pressure on the contact surface in supine and prone postures would influence data acquisition and lower the CI and RMSE values. Configuration $\mathrm{D}$ is not sensitive to the change of body posture and oppression associated with left lying and right lying, and the CI and RMSE values are consistent for all the body postures. The protocol of this study does not assess the effect of motion. Ensemble averaging techniques and adaptive filter algorithms to attenuate physiological noise [18, 33-35] may provide clearer understanding of the effect of different configurations. In the future work, more subjects, including women and the elderly, should be tested to further validate the robustness of different electrode configurations. Future studies can also focus on using finite element analysis to investigate the relationship between the distribution of electric field and the different configurations.

\section{CONCLUSION}

The results of this study provide experimental support for applying the electrodes in configuration D for long-term respiratory monitoring, where the electrodes are placed on intersections of left and right axillary midline with the fifth and seventh ribs, respectively. In such arrangement, the IP signal is least affected by noise, respiratory pattern, and body posture, and there is high agreement between the IP and IPNT signals. 


\section{ACKNOWLEDGEMENTS}

This work was supported by grants from the Guangdong Provincial Natural Science Foundation (S2012010010350) and the Guangdong Provincial Sensing Technology and Biomedical Equipment Laboratory (Project 2011A060901013), P. R. China.

\section{CONFLICT OF INTEREST}

The authors indicate no potential conflicts of interest.

\section{REFERENCES}

[1] Folke M, Cernerud L, Ekström M, Hök B. Critical review of non-invasive respiratory monitoring in medical care. Medical and Biological Engineering and Computing, 2003, 41(4):377-383.

[2] Houtveen JH, Groot PF, de Geus EJ. Validation of the thoracic impedance derived respiratory signals using multilevel analysis. International Journal of Psychophysiology, 2006, 59(2):97-106.

[3] Koivumäki T, Vauhkonen M, Kuikka JT, Hakulinen MA. Optimizing bioimpedance measurement configuration for dual-gated nuclear medicine imaging: A sensitivity study. Medical \& biological engineering \& computing, 2011, 49(7):783-791.

[4] Luo S, Afonso VX, Webster JG, Tompkins WJ. The electrode system in impedance-based ventilation measurement. Biomedical Engineering, IEEE Transactions on, 1992, 39(11):1130-1141.

[5] Peroni DG, Bodini A, Loiacono A, Paida G, Tenero L, Piacentini GL. Bioimpedance monitoring of airway inflammation in asthmatic allergic children. Allergologia et Immunopathologia, 2009, 37(1):3-6.

[6] Veiga J, Lopes AJ, Jansen JM, Melo PL. Fluctuation analysis of respiratory impedance waveform in asthmatic patients: effect of airway obstruction. Medical \& Biological Engineering \& Computing, 2012, 50(12):1249-1259.

[7] Williams EM, Powell T, Eriksen M, Neill P, Colasanti R. A pilot study quantifying the shape of tidal breathing waveforms using centroids in health and COPD. Journal of clinical monitoring and computing, 2013:1-8.

[8] Mlyńczak M, Cybulski G. Impedance pneumography: Is it possible? Photonics Applications in Astronomy, Communications, Industry, and High-Energy Physics Experiments 2012. International Society for Optics and Photonics, 2012, 84541T-84541T-14.

[9] Seppä VP, Pelkonen AS, Kotaniemi-Syrjänen A, Mäkelä MJ, Viik J, Malmberg LP. Tidal breathing flow measurement in awake young children by using impedance pneumography. Journal of Applied Physiology, 2013, 115(11):1725-1731.

[10] Krivoshei A, Kukk V, Min M. Decomposition method of an electrical bio-impedance signals into cardiac and respiratory components. Physiological measurement, 2008, 29(6):S15-S25.

[11] Lozano A, Rosell J, Pallas-Areny R. Errors in prolonged electrical impedance measurements due to electrode repositioning and postural changes. Physiological measurement, 1995, 16(2):121-130.

[12] Bayford R, Tizzard A. Bioimpedance imaging: an overview of potential clinical applications. Analyst, 2012, 137(20): 4635-4643.

[13] Lanatà, Scilingo EP, Nardini E, Loriga G, Paradiso R, De-Rossi D. Comparative evaluation of susceptibility to motion artifact in different wearable systems for monitoring respiratory rate. Information Technology in Biomedicine, IEEE Transactions on, 2010, 14(2):378-386.

[14] Lahtinen O, Seppä VP, Väisänen J, Hyttinen J. Optimal electrode configurations for impedance pneumography during sports activities. In 4th European Conference of the International Federation for Medical and Biological Engineering, Springer Berlin Heidelberg, 2009, 1750-1753.

[15] Koivumäki T, Vauhkonen M, Kuikka JT, Hakulinen MA. Bioimpedance-based measurement method for simultaneous acquisition of respiratory and cardiac gating signals. Physiological measurement, 2012, 33(8):1323-1334. 
[16] Carry P, Baconnier P, Eberhard A, Cotte P, Benchetrit G. Evaluation of respiratory inductive plethysmography: Accuracy for analysis of respiratory waveforms. Chest, 1997, 111(4):910-915.

[17] Eberhard A, Calabrese P, Baconnier P, Benchetrit G. Comparison between the respiratory inductance plethysmography signal derivative and the airflow signal. Frontiers in Modeling and Control of Breathing. Springer US, 2001: 489-494.

[18] Seppä VP, Viik J, Hyttinen J. Assessment of pulmonary flow using impedance pneumography. Biomedical Engineering, IEEE Transactions on, 2010, 57(9):2277-2285.

[19] Wang Q, Wang HB, Xu H, Zhou W, Liu GZ. A noninvasive urination-desire sensing method based on bladder bioimpedance spectrum analysis. Articles in Press, Journal of Medical Biological Engineering, (Sep 12, 2013).

[20] Urooj S, Khan M, Ansari AQ, Salhan AK. Measurement of thoracic impedance and approximations: a diagnosis technique for clinical utilization. Indian Journal of Industrial and Applied Mathematics, 2012, 3(2): 85-93.

[21] Cazalla O, Sebastiàn E, Cultrone G, Nechar M, Bagur MG. Three-way ANOVA interaction analysis and ultrasonic testing to evaluate air lime mortars used in cultural heritage conservation projects. Cement and Concrete Research, 1999, 29(11):1749-1752.

[22] Khalafalla AS, Stackhouse SP, Schmitt OH. Thoracic impedance gradient with respect to breathing. Biomedical Engineering, IEEE Transactions on, 1970 (3): 191-198.

[23] Lee H, Rusin CG, Lake DE, Clark MT, Guin L, Smoot TJ, Paget-Brown AO, Vergales BD, Kattwinkel J, Moorman JR, Delos JB. A new algorithm for detecting central apnea in neonates. Physiological measurement, 2012, 33(1):1-17.

[24] Adler J, Parmryd I. Quantifying colocalization by correlation: The Pearson correlation coefficient is superior to the Mander's overlap coefficient. Cytometry Part A, 2010, 77(8):733-742.

[25] Yu MC, Liou JL, Kuo SW, Lee MS, Hung YP. Noncontact respiratory measurement of volume changes using depth camera. Engineering in Medicine and Biology Society (EMBC), 2012 Annual International Conference of the IEEE, 2012, 2371-2374.

[26] Liu SP, Gao RX, He QB, Staudenmayer J, Freedson P. Development of statistical regression models for ventilation estimation. 31st Annual International Conference of the IEEE EMBS, 2009, 1266-1269.

[27] Seppänen TM, Alho OP, Seppänen T. Reducing the airflow waveform distortions from breathing style and body position with improved calibration of respiratory effort belts. Biomedical engineering online, 2013, 12(1): 97-114.

[28] Hatlestad JD, Moon LB, Hartley JW, Stahmann JE. Calibration of impedance monitoring of respiratory volumes using thoracic DC impedance: U.S. Patent 8,200,326. 2012.

[29] Seppä VP, Hyttinen J, Uitto M, Chrapek W, Viik J. Novel electrode configuration for highly linear impedance pneumography. Biomedizinische Technik/Biomedical Engineering, 2013, 58(1): 35-38.

[30] Grenvik A, Ballou S, McGinley E, Millen JE, Cooley WL, Safar P. Impedance Pneumography Comparison between Chest Impedance Changes and Respiratory Volumes in II Healthy Volunteers. Chest, 1972, 62(4): 439-443.

[31] Logic JL, Maksud MG, Hamilton LH. Factors affecting transthoracic impedance signals used to measure breathing. Journal of applied physiology, 1967, 22(2): 251-254.

[32] Perchiazzi G, Rylander C, Vena A, Derosa S, Polieri D, Fiore T, Hedenstierna G. Lung regional stress and strain as a function of posture and ventilatory mode. Journal of applied physiology, 2011, 110(5): 1374-1383.

[33] Hanifi A, Goplen N, Matin M, Salters R, Alam R. A linear parametric approach for analysis of mouse respiratory impedance. Biomedical Circuits and Systems, IEEE Transactions on, 2012, 6(3):287-294.

[34] Sankar AB, Kumar D, Seethalakshmi K. Performance study of various adaptive filter algorithms for noise cancellation in respiratory signals. Signal processing: An international journal, 2010, 4(5):267-278.

[35] Saatci E, Akan A. Respiratory parameter estimation in non-invasive ventilation based on generalized Gaussian noise models. Signal processing: An international journal, 2010, 90(2): 480-489. 



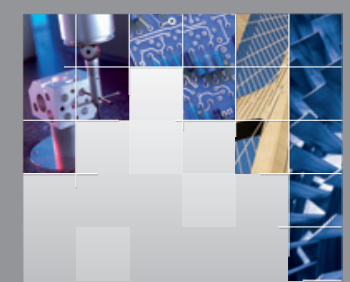

\section{Enfincering}
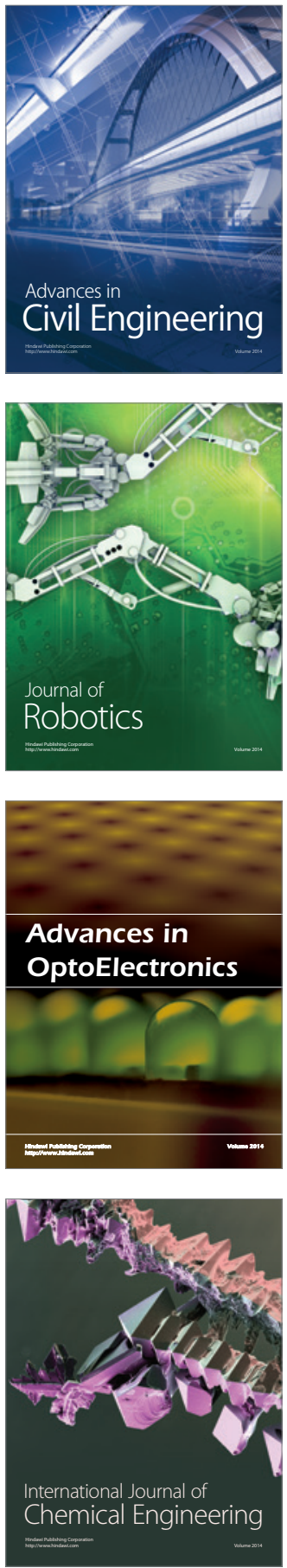

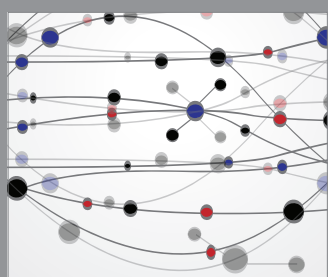

The Scientific World Journal

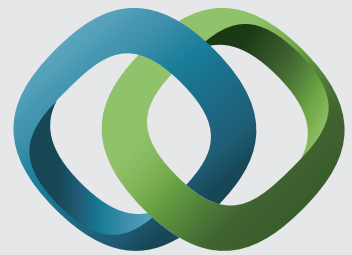

\section{Hindawi}

Submit your manuscripts at

http://www.hindawi.com
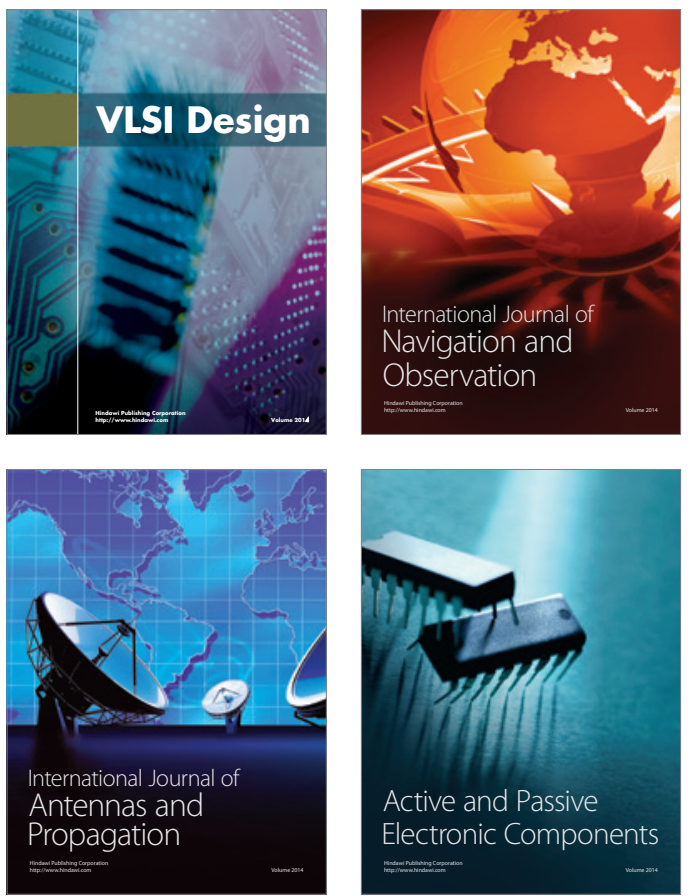
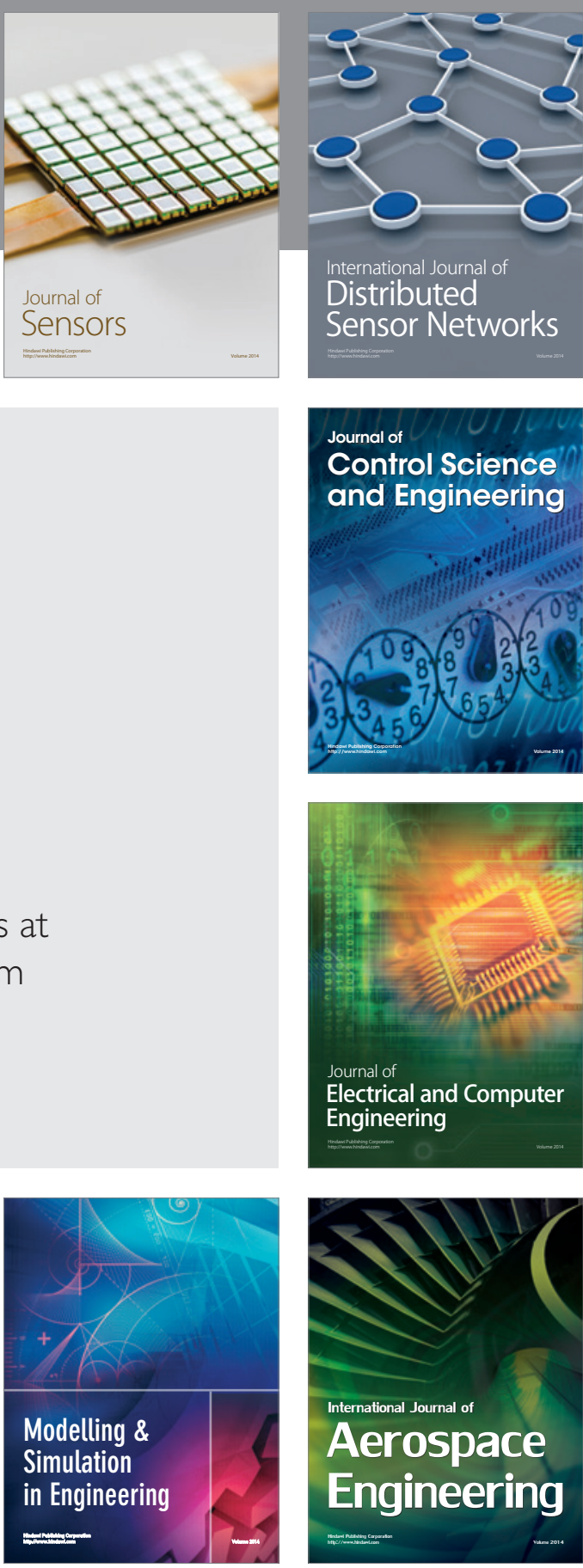

International Journal of

Distributed

Sensor Networks

Journal of

Control Science

and Engineering
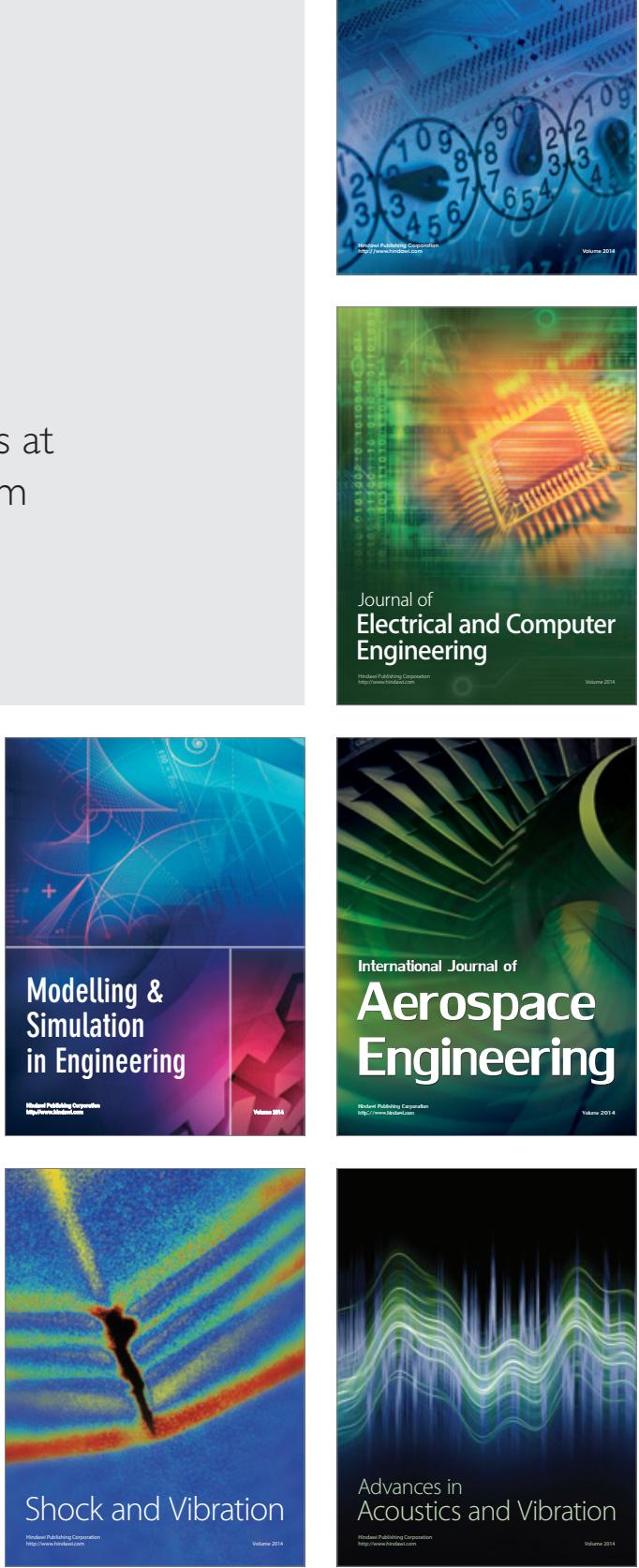